\title{
Onboard and Real-Time Artificial Satellite Orbit Determination Using GPS
}

\author{
Ana Paula Marins Chiaradia, ${ }^{1}$ Hélio Koiti Kuga, ${ }^{2}$ \\ and Antonio Fernando Bertachini de Almeida Prado ${ }^{2}$ \\ ${ }^{1}$ Grupo de Dinâmica Orbital e Planetologia, FEG/UNESP, 12 516-410 Guaratinguetá, SP, Brazil \\ ${ }^{2}$ Division of Space Mechanics and Control-INPE, Avenida dos Astronautas, 1758, 12 227-010 São José dos Campos, SP, Brazil
}

Correspondence should be addressed to Ana Paula Marins Chiaradia; anachiaradia@feg.unesp.br

Received 21 September 2012; Accepted 9 January 2013

Academic Editor: Maria Zanardi

Copyright (C) 2013 Ana Paula Marins Chiaradia et al. This is an open access article distributed under the Creative Commons Attribution License, which permits unrestricted use, distribution, and reproduction in any medium, provided the original work is properly cited.

\begin{abstract}
An algorithm for real-time and onboard orbit determination applying the Extended Kalman Filter (EKF) method is developed. Aiming at a very simple and still fairly accurate orbit determination, an analysis is performed to ascertain an adequacy of modeling complexity versus accuracy. The minimum set of to-be-estimated states to reach the level of accuracy of tens of meters is found to have at least the position, velocity, and user clock offset components. The dynamical model is assessed through several tests, covering force model, numerical integration scheme and step size, and simplified variational equations. The measurement model includes only relevant effects to the order of meters. The EKF method is chosen to be the simplest real-time estimation algorithm with adequate tuning of its parameters. In the developed procedure, the obtained position and velocity errors along a day vary from 15 to $20 \mathrm{~m}$ and from 0.014 to $0.018 \mathrm{~m} / \mathrm{s}$, respectively, with standard deviation from 6 to $10 \mathrm{~m}$ and from 0.006 to $0.008 \mathrm{~m} / \mathrm{s}$, respectively, with the SA either on or off. The results, as well as analysis of the final adopted models used, are presented in this work.
\end{abstract}

\section{Introduction}

The Global Positioning System (GPS) is a satellite navigation system that has been used to determine the position, velocity, and time with high accuracy of an artificial satellite, such as TOPEX/POSEIDON (T/P) [1], Jason-1, Jason-2, CHAMP, and GRACE satellites. Current samples of satellites having an onboard GPS receiver are Jason [2-4], GRACE [5, 6], CHAMP $[7,8]$, and KOMPSAT $[9,10]$.

The TOPEX/POSEIDON (T/P) mission is jointly conducted by the United States National Aeronautics and Space Administration (NASA) and the French Space Agency, Centre National d'Etudes Spatiales (CNES). The main goal of this mission is to improve the knowledge of the global ocean circulation. Other applications include the ocean tides, geodesy and geodynamics, ocean wave height, and wind speed [1]. The T/P spacecraft orbits the Earth at an altitude of $1336 \mathrm{~km}$, inclination of $66^{\circ}$, and near-zero eccentricity. The period of the orbit is $1.87 \mathrm{hr}$. The satellite orbit must be determined with an RMS radial accuracy of $13 \mathrm{~cm}$. This is an extremely stringent accuracy requirement for a satellite of this shape and altitude [11]. The T/P GPS receiver can track up to 6 GPS satellites at once in both frequencies if antispoofing is inactive.

Its successor, Jason-1, continues the time series of centimeter-level ocean topography observations as it follows on to the highly successful TOPEX/Poseidon (T/P) radar altimeter satellite. Jason has the same orbital parameters of T/P satellite. Preliminary tests of orbits computed using Jason-1 tracking data in a reduced dynamic strategy suggest that the RMS radial accuracies are already better than $2 \mathrm{~cm}$, and that the $1 \mathrm{~cm}$ goal is within reach [2].

The GRACE (Gravity Recovery and Climate Experiment) satellites, launched in March 2002 with about $470 \mathrm{~km}$ in altitude, are each equipped with a BlackJack GPS onboard receiver for precise orbit determination and gravity field recovery [5]. With primary objective of determining the Earth's gravity field and its temporal variations with unprecedented accuracy, accurate orbits for GRACE are required. The orbit determination has been obtained with 
accuracy better than $5 \mathrm{~cm}$ in each direction for GRACE orbits. The relative accuracy of the two GRACE satellites is about $1 \mathrm{~cm}$ in position and 10 micrometers per second in velocity.

The CHAllenging Minisatellite Payload (CHAMP) is a German small satellite used for geophysical research and application. This mission is scheduled to last five years in order to provide a sufficiently long observation time to resolve long-term temporal variations primarily in the magnetic field, in the gravity field, and within the atmosphere. CHAMP was launched on 15 July 2000 and reentered the Earth's atmosphere on September 20, 2010 [12]. The position accuracy is about $0.8 \mathrm{~m}$ for pseudorange in single frequency [13].

Two types of orbit determination are performed at the KOMPSAT using GPS data. One is the operational orbit determination using GPS navigation solutions providing the position accuracy around $4.45 \mathrm{~m}$ RMS and the velocity accuracy around $0.005 \mathrm{~m} / \mathrm{s}$ RMS. The other is the precise orbit determination using GPS raw measurement data by DGPS technique. In this case, the difference between NASA GSFC Precise Orbit Ephemeris and precise orbit determination results using TEC are radial $6.9 \mathrm{~cm}$ RMS, along track $19.4 \mathrm{~cm}$ RMS, and cross track $6.9 \mathrm{~cm}$ RMS [8].

Gomes et al. [14] analyzed a real-time orbit estimator using the raw navigation solution provided by GPS receivers. The estimation algorithm considers a Kalman filter with a rather simple orbit dynamic model and random walk modeling of the receiver clock bias and drift. Using the Topex/Poseidon satellite as test bed, characteristics of model truncation, sampling rates, and degradation of the GPS receiver (Selective Availability) were analyzed. The position precision obtained was around $20-30 \mathrm{~m}$ in $3 \mathrm{D}$ and around $0.5 \mathrm{~m} / \mathrm{s}$ in velocity.

Pardal et al. [15] determined the orbit of an artificial satellite and analyzed its implications, using least squares algorithms through sequential given rotations as the method of estimation, and data of the GPS receivers. This approach has the goal of improving the performance of the orbit estimation process and, at the same time, of minimizing the computational procedure cost. Perturbations due to high order geopotential and direct solar radiation pressure and the position of the GPS antenna on the satellite body were taken into account.

In this paper, an algorithm to determine onboard the satellite orbit in real-time using the GPS system and Kalman filtering is developed. It used a simplified and compact model with low computational cost. The extended Kalman filter (EKF) estimates the state vector, composed of the position and velocity components, bias, drift, and drift rate of the GPS receiver clock. A simple fixed step-size-fourth order RungeKutta numerical integrator is found to be suitable to integrate the differential equations of the orbital motion. The force model in the equations of motion may consider the perturbations due to the geopotential up to any order and degree of the spherical harmonics. The state error covariance matrix is computed through the transition matrix, which is calculated analytically in an optimized way. The raw single frequency pseudorange GPS measurements are used as observations by the Kalman filter. They are modeled taking into account most of the GPS satellite and receiver clock offsets. The main aim is to develop a GPS-based real-time orbit determination system for any artificial Earth satellite. The target is orbit accuracy in the order of tens of meters with simple modeling of the dynamics or measurement systems, and still keeping low requirements of speed and computational burden.

\section{Orbit Determination}

The orbit determination process consists of obtaining values of the parameters that completely specify the motion of an orbiting body, like artificial satellites, based on a set of observations of the body. It involves nonlinear dynamical and nonlinear measurement systems, which depends on the tracking system and estimation technique (e.g., Kalman filtering or least squares [16-21]). The dynamical system model consists of orbital motion dynamics, measurements models, Earth's rotation effects, and perturbation models. As Montenbruck and Gill [22], besides the state variables that define the initial conditions, these models depend on a variety of parameters that affects either the dynamical motion or the measurement process. Due to the complexity of the applied models, it is hardly possible to solve directly for any of these parameters from a given set of observations.

The observation may be obtained from the ground station networks using laser, radar, Doppler, and so forth, or by space navigation systems, as the Global Positioning System (GPS). The choice of the tracking system depends on a compromise between the goals of the mission and the tools available.

In the case of the GPS, the advantages are global coverage, high precision, low cost, and autonomous navigation resources. The GPS may provide orbit determination with accuracy at least as good as methods using ground-tracking networks. The latter provides standard precision around hundred meters and the former can provide precision as tight as some centimeters.

The GPS provides, at a given instant, a set of many redundant measurements, which makes the orbit position observable geometrically.

After some advances of technology, the single frequency GPS receivers provide a good basis to achieve fair precision at relatively low cost, still attaining the accuracy requirements of the mission.

The GPS allows the receiver to determine its position and time geometrically anywhere at any instant with data from at least four satellites. The principle of navigation by satellites is based in sending signals and data from the GPS satellites to a receiver that is inside the satellite that needs to have its orbit determined. This receiver measures the travel time of the signal and then calculates the distance between the receiver and the GPS satellite. If the clocks are synchronized, measurements from three GPS satellites are enough to obtain its position. If the clocks are not synchronized, four measurements are required. Those measurements of distances are called pseudorange, as shown in Figure 1. 




Figure 1: Orbit determination using GPS.

\section{Description of the Algorithm}

The algorithm is developed to determine artificial satellite orbits according the dynamical model (the force model). The method is used to integrate the differential equations of a satellite motion, the computation of the state transition matrix, the measurement model, and the estimation technique.

For that sake, the following aspects are verified:

(a) concerning the dynamical model: what is better order and degree truncation of the spherical harmonic coefficients of the JGM-2 model in order to reach the objectives in terms of low computational cost, relative accuracy (tens of meters), and the lowest possible order and degree of the harmonical coefficients;

(b) concerning the integration method: with step size of the fixed step RK4 integrator to use, considering low computational cost, accuracy, and the selection of appropriate reference systems and transformations;

(c) considering the measurement model: where the errors source is included in the model; and

(d) considering the filtering: the EKF is used and the use of the simplified transition matrix considering either the pure Keplerian motion or including at least the $J_{2}$ effects.

3.1. Force Model and Numerical Integration. The main forces acting on an artificial satellite may be classified as gravitational (e.g., geopotential, third-body perturbations, and tides) and nongravitational forces (e.g., atmospheric drag, solar radiation pressure). These forces are rather difficult to model, so a mathematically inaccurate model of the motion with respect to true motion is sometimes adopted in Kuga $[23,24]$.

To choose a simplified force model to be adopted in this work, the following factors are considered: generality, orbit accuracy, and computational cost for orbit determination in a real-time and onboard environment. Therefore, only forces due to the Earth gravitational field should be implemented.

The considered harmonic coefficients of the Earth geopotential field are set up to order and degree 10 of JGM-2 model, according to studies developed in Chiaradia et al. [25], without overloading the processing time.

The acceleration and the related partial derivatives matrices are computed through the recurrence relations, according to Pines [26], in Earth-fixed (EF) coordinates. The coordinate transformation from True of Date (ToD) to Pseudo-Earth fixed equator and prime meridian (PEF) takes into account the Earth sidereal rotation, but the polar motion is neglected in this work.

The integration of the satellite motion equation is carried out using the simple 4th order of the Runge-Kutta (RK4) algorithm. It is implemented without any mechanism of step adjustment or error control. The RK4 is considered an adequate numerical integrator, due to its simplicity, fair accuracy, low truncation error, and low computational burden. An initialization procedure is not necessary and the step size is quite easy to change. The 30-second integration step interval is used.

3.2. Measurement Model. With the advances of technology, the single frequency GPS receivers provide a good basis to achieve fair precision at a relatively low cost, still attaining the accuracy requirements of general missions. In this way, only the code pseudorange in L1 frequency is used to determine the satellite orbit, providing the accuracy required by the mission, similar to the one shown in this work.

The errors considered in the $\mathrm{C} / \mathrm{A}$ code pseudorange measurements are the GPS satellite and receiver clock offsets. Chiaradia et al. $[25,27]$ studied the need of considering the ionospheric correction model. It has been concluded that the ionospheric effect (around 3-6 meters) does not affect significantly the final accuracy of the orbit. In this way, it will be neglected once the goal is not to achieve high precision (around centimeters). In high precision and onboard applications, some ionospheric correction for single frequency should be applied, but this may add some complexity if we need to implement a better ionospheric model.

The equation of the C/A code pseudorange in L1 frequency is given by

$$
\rho_{c}=\rho+c\left[\Delta t_{\mathrm{GPS}}(t)-\Delta t_{u}(t)\right],
$$

where $\rho_{c}$ is the C/A code pseudorange in L1, $c$ is the speed of light, $\Delta t_{\mathrm{GPS}}$ is the GPS satellite clock offset, $\Delta t_{u}$ is the receiver clock offset, $t$ is the observation instant in GPS time, and $\rho$ is the geometric range given by

$$
\rho=\sqrt{\left(x_{\mathrm{GPS}}-x\right)^{2}+\left(y_{\mathrm{GPS}}-y\right)^{2}+\left(z_{\mathrm{GPS}}-z\right)^{2}},
$$

where $x, y$, and $z$ are the positional states of the user satellite at reception time (in GPS time) and $x_{\mathrm{GPS}}, y_{\mathrm{GPS}}$, and $z_{\mathrm{GPS}}$ are 
the positional states of the GPS satellite at transmission time (in GPS time), corrected for light time delay.

The second term on the right side of (1) is the clock bias that represents the combined clock offsets of the satellite and of the receiver with respect to GPS time. Each GPS satellite contributes with one clock bias. The information for GPS satellite clocks is known and transmitted via a navigation message in the form of three polynomial coefficients with a reference time $t_{\mathrm{oc}}$. The clock correction of the GPS satellite for epoch $t$ is

$$
\begin{gathered}
t=t_{\mathrm{GPS}}-\Delta t_{\mathrm{GPS}}, \\
\Delta t_{\mathrm{GPS}}=a_{f 0}+a_{f 1}\left(t-t_{\mathrm{oc}}\right)+a_{f 2}\left(t-t_{\mathrm{oc}}\right)^{2}+\delta_{R}^{\mathrm{GPS}}, \\
\delta_{R}^{\mathrm{GPS}}=\frac{2}{c^{2}} \sqrt{a \mu} e \sin E,
\end{gathered}
$$

where $t_{\mathrm{GPS}}$ is the spacecraft code phase time at message transmission time in seconds; $a_{f 0}, a_{f 1}$, and $a_{f 2}$ are bias, drift, and drift rate of GPS satellite clock, respectively; $t_{\mathrm{oc}}$ is the reference time, in seconds, measured from the GPS time weekly epoch; $\delta_{R}$ is a small relativistic correction of the GPS satellite clock caused by orbital eccentricity $e$; $a$ is the semimajor axis of the orbit; $\mu$ is the Earth gravitational constant; and $E$ is the eccentric anomaly.

The relativistic effect is included in the clock polynomial broadcast via navigation message. If a more accurate equation is required, the relativistic effects must be subtracted from the clock polynomial coefficients. The polynomial coefficient $a_{f 0}$, $a_{f 1}$, and $a_{f 2}$ are transmitted in units of sec, nondimensional, and $1 / \mathrm{sec}$, respectively. The clock data reference time $t_{\mathrm{oc}}$ is also broadcasted. The value of $t_{\mathrm{GPS}}$ must account for the beginning or the end-of-week crossovers. The user may approximate $t$ by $t_{\mathrm{GPS}}$ in (3).

The user clock offset is part of the estimated state vector given by [28]

$$
c \Delta t_{u}=b_{0}+b_{1} \Delta T+b_{2} \Delta T^{2}+\delta_{R}^{\mathrm{USER}}
$$

where $b_{0}, b_{1}$, and $b_{2}$ are the bias, drift, and drift rate of the user clock and $\Delta T$ is the elapsed time since the instant of the first measurement. The relativistic effect in the user clock is calculated by using the best available estimated state vector in the epoch.

3.3. GPS Travel and Reception Times. The computation of geometric range $\rho$ is required for both pseudorange and carrier phase processing. The GPS position coordinates are used at the instant of emission. However, the reception time is used to compute it. Then, this time is corrected by subtracting the signal travel time to obtain the emission time. Therefore, the travel time is computed through an iterative process that starts assuming an average value for travel time $\tau$. Next, the GPS position for the epoch $(t-\tau)$ is interpolated and then the geometric range is computed, which can be used to reconstruct the travel time by [29]

$$
\tau=\frac{\rho}{c}
$$

The light-time iteration is usually performed in an inertial system with position vector $\mathbf{r}=(x, y, z)$ and the GPS satellite position vector $\mathbf{r}_{\mathrm{GPS}}=\left(x_{\mathrm{GPS}}, y_{\mathrm{GPS}}, z_{\mathrm{GPS}}\right)$. Then, the positions from inertial to the Earth-fixed WGS84 system or vice versa are transformed. So, the signal path is given as [22]

$$
\rho=\mathbf{R}^{\mathrm{WGS}}(t)\left(\mathbf{r}_{\mathrm{GPS}}(t-\tau)-\mathbf{r}(t)\right),
$$

where $\mathbf{R}^{\text {WGS }}$ is the rotation matrix from inertial to Earth-fixed WGS-84 system. Making use of the approximation

$$
\mathbf{R}^{\mathrm{WGS}}(t) \approx \mathbf{R}_{z}\left(\omega_{e} \tau\right) \mathbf{R}^{\mathrm{WGS}}(t-\tau)
$$

where $\omega_{e}$ is the Earth rotation rate, the inertial position of the GPS satellite may be substituted by the corresponding Earthfixed position:

$$
\mathbf{r}_{\mathrm{GPS}}^{\mathrm{WGS}}(t-\tau)=\mathbf{R}^{\mathrm{WGS}}(t-\tau) \mathbf{r}_{\mathrm{GPS}}(t-\tau) .
$$

This yields in the Earth-fixed reference frame

$$
\rho=c \tau=\left|\mathbf{R}_{z}\left(\omega_{e} \tau\right) \mathbf{r}_{\mathrm{GPS}}(t-\tau)-\mathbf{r}^{\mathrm{WGS}}(t)\right| .
$$

If the discrepancy between the first and second approximation of $\tau$ is greater than a specified criterion, the iteration is repeated; that is, a new satellite position is interpolated and a new distance is computed and so on. Generally, a couple of iterations are sufficient.

This computation is not affected by the receiver clock error $\Delta t_{u}$ and the satellite clock error $\Delta t_{s v}$. However, the ionosphere troposphere, hardware, and multipath corrupt the computed nominal emission time. All of these effects are neglected in the present context. The same process corrects the reception time as well.

3.4. Estimation Technique. The standard Kalman filter algorithm is used to estimate the spacecraft orbit onboard. This filter is robust, with easy implementation for applications in real-time and no requirement of iterating the previously collected data, and is able to provide the current orbit in real-time. The Extended Kalman Filter (EKF) is a version which is applicable to nonlinear problems, and it is composed of time-update and measurement-update cycles. The timeupdate phase updates the state and the covariance matrix along the time using dynamical equations. In this work, the estimated state vector is given by

$$
\mathbf{x}=(\mathbf{r}, \mathbf{v}, \mathbf{b})^{T},
$$

where $\mathbf{r}=(x, y, z)^{T}$ and $\mathbf{v}=(\dot{x}, \dot{y}, \dot{z})^{T}$ are the spacecraft's position and velocity vectors, $\mathbf{b}=\left(b_{0}, b_{1}, b_{2}\right)^{T}$, where $b_{0}, b_{1}$, and $b_{2}$ are bias, drift, and drift rate of the receiver clock, respectively, and $t$ is the instant of integration. All coordinates refer to ToD system.

The algorithm used can be found in references [18, 19]. The details of how it is implemented here can be found in reference [30].

The state error covariance matrix is propagated through the transition matrix. The conventional filter is implemented 


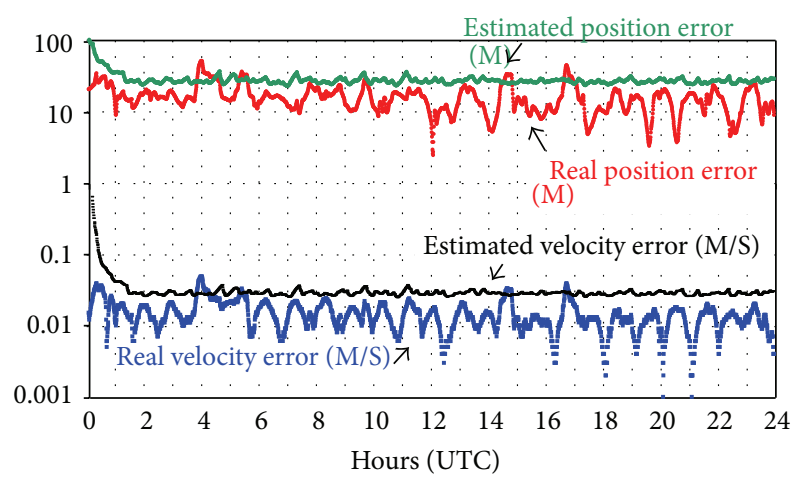

FIGURE 2: Estimated and real error with SA off.

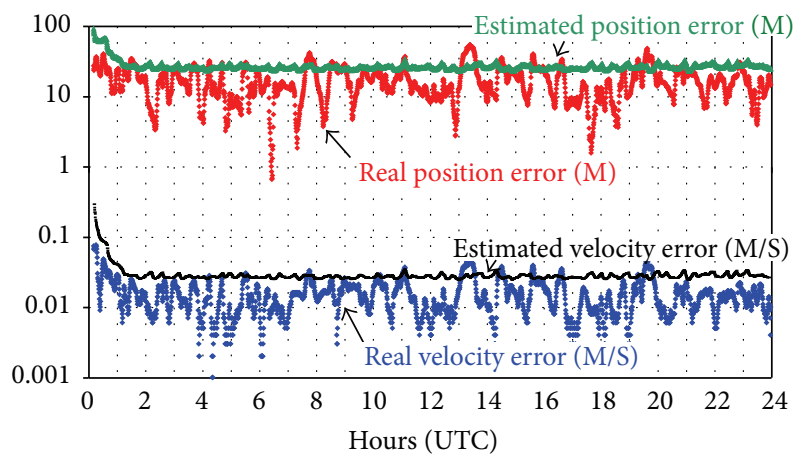

FIGURE 3: Estimated and real error with SA on.

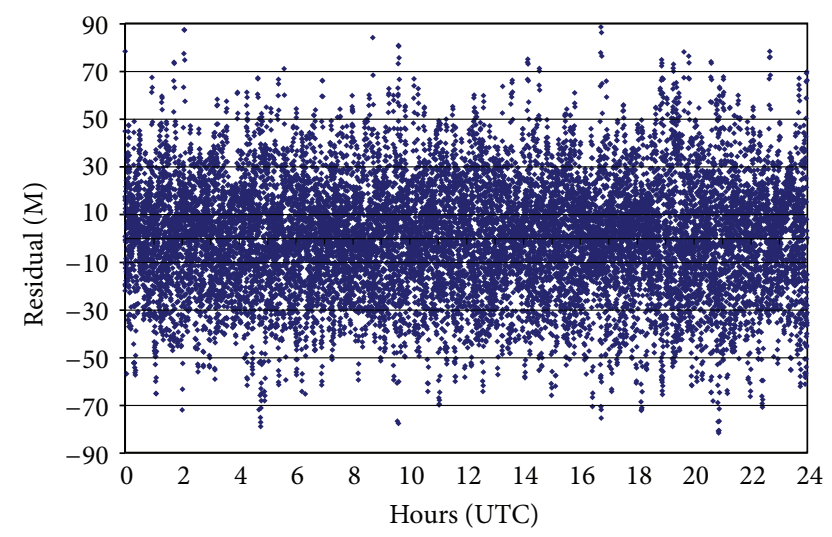

FIGURE 4: Typical behavior of the residuals.

by calculating only the upper triangular part of the state error covariance matrix. The other elements are obtained imposing symmetry to the matrix. The lack of symmetry is one of the highest sources of truncation error per Kalman filter cycle [31].

One method to avoid the problem of high computational cost and extended analytical expressions of the transition matrix consists of propagating the state vector using the complete force model and then, computing the transition matrix using a simplified force model. The analytical calculation of the transition matrix of the Keplerian motion is a reasonable approximation when only short time intervals of the observations and reference instant are involved [31]. On the other hand, the inclusion of $J_{2}$ (Earth flattening) effect in the transition matrix can approximately be done by adopting the Markley's method [32].

Chiaradia et al. [33] studied these two methods. The first method is an approximation of the Keplerian motion, providing an analytical solution, which is then calculated numerically by solving the Kepler's equation. The second one is a local numerical approximation that includes the effect of $J_{2}$. They were evaluated according to accuracy, processing time, and handling complexity of the equations for two kinds of orbits. It has been concluded that the Keplerian motion model, which was developed by Kuga [31], is still a better suited force model to be adopted in the evaluation of a general-purpose state transition matrix. Some other papers studying similar or related topics can be found in references [34-36].

\section{Simulations}

To validate and to analyze the algorithm, the Topex/Poseidon $(\mathrm{T} / \mathrm{P})$ satellite data is used. This satellite carries a dual frequency receiver GPS onboard to experimentally test the ability of the GPS to provide Precise Orbit Determination (POD). The following files are used:

(i) the T/P observation files that broadcast the code and carrier pseudorange measurements in two frequencies in 10-second GPS time steps and are provided by the GPS Data Processing Facility of the Jet Propulsion Laboratory (JPL) in RINEX format;

(ii) the T/P Precise Orbit Ephemeris (POE) files that are generated by JPL; and

(iii) the broadcast GPS navigation message file in RINEX format provided by Crystal Dynamics Data Information System (CDDIS) of the Goddard Space Flight Center (GSFC).

The estimated position and velocity are compared against the T/P-POE. It is claimed to estimate the T/P position with accuracy, which is better than $15 \mathrm{~cm}$. The states in the POE are provided in one minute UTC time steps in ToD system. The T/P GPS measurements are provided in 10 seconds of GPS time. According to IERS, the difference between the UTC and GPS times is approximately an integer number of seconds, increasing timely with the introduction of leap seconds. For example, the difference is 9 seconds for 1993.

However, in the process of orbit determination, the state is estimated in 30 -second intervals in UTC time with transmission and reception time corrections. Then, these data instants are not coincident. Therefore, the states are interpolated through an interpolation (Polint) subroutine [37]. With this approach, the mean error of the interpolated states is $0.068 \mathrm{~m}$ and $2.5 \times 10^{-4} \mathrm{~m} / \mathrm{s}$ for position and velocity, respectively, which does not add any significant bias to the accuracy evaluation of the results. 


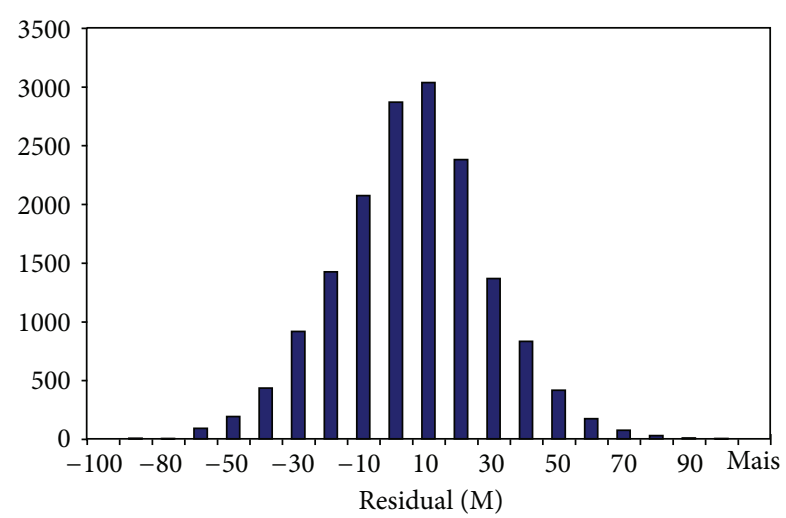

FIgURE 5: Histogram of the residuals.

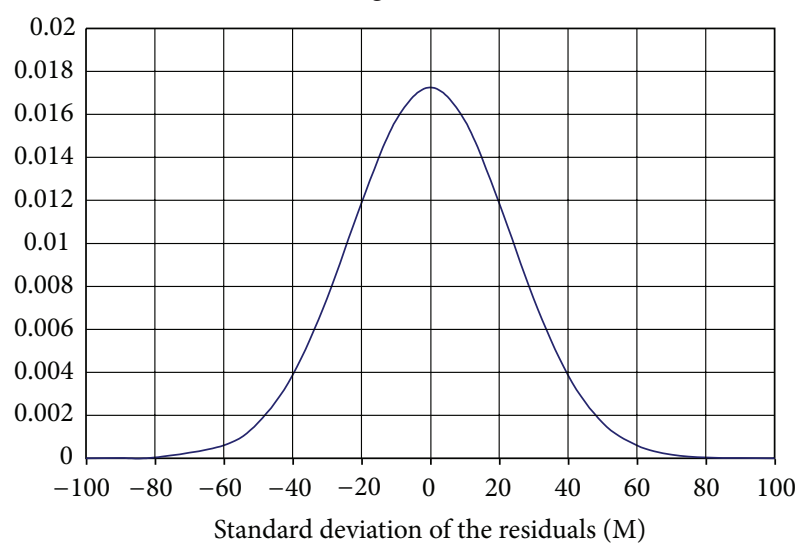

FIGURE 6: Normal distribution of the residuals.

Some parameters to be used in evaluating the algorithm in the tests are defined. The real position error is given by

$$
\Delta \mathbf{r} \equiv\left[\sum_{i=1}^{3}\left(x_{i}-\widehat{x}_{i}\right)^{2}\right]^{1 / 2},
$$

where $x_{i}$ and $\widehat{x}_{i}, i=1,2,3$, are the reference components (or real) and estimated position vectors, respectively. The estimated position error is given by

$$
\Delta \widehat{\mathbf{r}}=\left[\sum_{i=1}^{3} \mathbf{P}_{i i}\right]^{1 / 2},
$$

where $\mathbf{P}_{i i}, i=1,2,3$, represents the values of the diagonal elements of the state covariance matrix of the estimated position. The real velocity error is given by

$$
\Delta \mathbf{v} \equiv\left[\sum_{i=4}^{6}\left(x_{i}-\widehat{x}_{i}\right)^{2}\right]^{1 / 2},
$$

where $x_{i}$ and $\hat{x}_{i}, i=4,5,6$, are the components of the reference (or real) and estimated velocity vectors, respectively. The estimated velocity error is given by

$$
\Delta \widehat{\mathbf{v}}=\left[\sum_{i=4}^{6} \mathbf{P}_{i i}\right]^{1 / 2},
$$

TABLE 1: Statistical errors with SA on or off.

\begin{tabular}{lccc}
\hline Date & $\Delta r(\mathrm{~m})$ & $\Delta v(\mathrm{~m})$ & Residuals $(\mathrm{m})$ \\
\hline $11 / 18 / 1993$ & $15.5 \pm 6.8$ & $0.014 \pm 0.006$ & $0.027 \pm 13.2$ \\
$11 / 19 / 1993$ & $17.4 \pm 6.7$ & $0.016 \pm 0.006$ & $-0.130 \pm 13.4$ \\
$11 / 20 / 1993$ & $17.6 \pm 8.4$ & $0.017 \pm 0.007$ & $-0.160 \pm 22.6$ \\
$01 / 03 / 1994$ & $16.5 \pm 8.5$ & $0.015 \pm 0.008$ & $0.004 \pm 22.1$ \\
$01 / 04 / 1994$ & $19.5 \pm 9.6$ & $0.018 \pm 0.009$ & $-0.160 \pm 22.8$ \\
$01 / 05 / 1994$ & $19.6 \pm 7.8$ & $0.018 \pm 0.008$ & $0.040 \pm 22.9$ \\
$01 / 21 / 1994$ & $19.0 \pm 7.7$ & $0.018 \pm 0.008$ & $-0.050 \pm 23.9$ \\
$01 / 22 / 1994$ & $16.5 \pm 8.6$ & $0.015 \pm 0.008$ & $-0.007 \pm 23.3$ \\
\hline
\end{tabular}

where $\mathbf{P}_{i i}, i=4,5,6$, represents the values of the diagonal elements of the state covariance matrix of the estimated velocity. And, the residual is given by:

$$
\Delta \boldsymbol{\rho}=\mathbf{z}-\boldsymbol{\rho}_{c},
$$

where $\mathbf{z}$ and $\boldsymbol{\rho}_{c}$ are the observed and calculated pseudorange measurements, respectively.

\section{Results}

The algorithm is analyzed and tested using 8 complete days of data. Figures 2 and 3 show the real and filtered position and velocity errors in $\mathrm{m}$ and $\mathrm{m} / \mathrm{s}$, with SA (Select Availability) off and on, respectively. These figures show the results for one day of filtering.

Figure 4 shows the typical behavior of the pseudorange residuals for all tests.

Figures 5 and 6 show the histogram and normal distribution of the residuals.

Table 1 shows the statistical error for position and velocity vectors and the residual for all tests.

It can be noted that, for all days tested, the real position and velocity errors are fewer than with the estimated position and velocity errors by the filter. It shows the conservative behavior of the filter. In all cases, the filtering takes around one hour to converge. Before achieving the convergence, the onboard computer can use the GPS navigation solution provided by GPS receiver with 100 -meter position error. The position accuracy with the SA off (11/18/1993 and 11/19/1993) or on (other days) is from 15 to $20 \mathrm{~m}$, with standard deviation from 6 to $10 \mathrm{~m}$. The velocity accuracy varies from 0.014 to $0.018 \mathrm{~m} / \mathrm{s}$, with standard deviation going from 0.006 to $0.009 \mathrm{~m} / \mathrm{s}$, as shown in Table 1 .

Through Figures 5 and 6 and Table 1, it can be noted that the residuals present normal distribution with mean zero and standard deviation of around $23 \mathrm{~m}$. On November 18 and 19, 1993, the SA is off and the standard deviation of the residuals is around $13.3 \mathrm{~m}$. This fact shows how the SA affects the statistics of the residuals, although the estimated state had not been significantly degraded.

\section{Conclusions}

The main goal of this work is to achieve accuracy around tens of meters along with minimum computational cost when 
determining, in real-time, artificial satellite orbits onboard considering a simplified model. For its development, single frequency GPS measurements are used, considering the effects of the clock offsets of the GPS and user satellites and user relativistic effects.

This algorithm is tested with real data from a satellite with a GPS receiver onboard. The satellite orbit is estimated using the developed algorithm with a good accuracy and minimum computational cost. The algorithm uses a large 30-second step size of propagation (10 seconds step size can be used as well), the geopotential model up to order and degree 10, and the computation method of the transition matrix considering the Keplerian motion. The position accuracy obtained is better than $20 \mathrm{~m}$ with the SA on or off. The results are according to expected.

\section{Acknowledgments}

The authors wish to express their appreciation for the support provided by UNESP (Universidade Estadual Paulista "Júlio de Mesquita Filho") of Brazil and INPE (Brazilian Institute for Space Research). They also wish to acknowledge the English language review performed by FDCT-Foundation for Scientific and Technological Development.

\section{References}

[1] L. L. Fu, E. J. Christensen, C. A. Yamarone Jr. et al., "TOPEX/ POSEIDON mission overview," Journal of Geophysical Research, vol. 99, no. 12, pp. 24369-24381, 1994.

[2] B. Haines, W. Bertiger, S. Desai et al., "Initial orbit determination results for Jason-1 towards a 1-cm orbit," in Proceedings of the Institute of Navigation Conference (GPS '02), Portland, Oregon, USA, September 2002.

[3] K. R. Choi, J. C. Ries, and B. D. Tapley, "JASON-1 precision orbit determination by combining SLR and DORIS with GPS tracking data," Marine Geodesy, vol. 27, no. 1-2, pp. 319-331, 2004.

[4] S. B. Luthcke, N. P. Zelensky, D. D. Rowlands, F. G. Lemoine, and T. A. Williams, "The 1-centimeter orbit: Jason-1 precision orbit determination using GPS, SLR, DORIS, and altimeter data," Marine Geodesy, vol. 26, no. 3-4, pp. 399-421, 2003.

[5] Z. Kang, P. Nagel, and R. Pastor, "Precise orbit determination for GRACE,” Advances in Space Research, vol. 31, no. 8, pp. 18751881, 2003.

[6] Z. Kang, B. Tapley, S. Bettadpur, J. Ries, P. Nagel, and R. Pastor, "Precise orbit determination for the GRACE mission using only GPS data," Journal of Geodesy, vol. 80, no. 6, pp. 322-331, 2006.

[7] D. Kuang, Y. Bar-Sever, W. Bertiger et al., "Precision orbit determination for CHAMP using GPS data from BlackJack receiver," in Proceeding of the ION National Technical Meeting, Long Beach, Calif, USA, January 2001.

[8] S. Zhu, C. Reigber, and R. Koenig, "Integrated adjustment of CHAMP, GRACE and GPS data," Journal of Geodesy, vol. 78, no. 1-2, pp. 103-108, 2004.

[9] B. S. Lee, J. S. Lee, J. H. Kim et al., "Reconstruction of KOMPSAT-1 GPS navigation solutions using GPS data generation and preprocessing program," Acta Astronautica, vol. 54, no. 8 , pp. 571-576, 2004.
[10] B. S. Lee, J. C. Yoon, Y. Hwang, and J. Kim, "Orbit determination system for the KOMPSAT-2 using GPS measurement data," Acta Astronautica, vol. 57, no. 9, pp. 747-753, 2005.

[11] B. H. Putney, J. A. Marshall, R. S. Nerem et al., "Precise orbit determination for the Topex/Poseidon mission," in Proceedings of the AAS/AIAA Astrodynamics Conference, pp. 195-212, August 1993.

[12] C. Noll, International Laser Ranging Service, 2012, http://ilrs .gsfc.nasa.gov/satellite_missions/list_of_satellites/cham_general .html.

[13] E. Gill and O. Montebruck, "Comparision of GPS-based orbit determination strategies," in Proceedings of the 18th International Symposium on Space Flight Dynamics, Munich, Germany, October 2004.

[14] V. M. Gomes, H. H. Kuga, and A. P. M. Chiaradia, "Real time orbit determination using GPS navigation solution," Journal of the Brazilian Society of Mechanical Sciences and Engineering, vol. 29, no. 3, pp. 274-278, 2007.

[15] P. C. P. M. Pardal, H. K. Kuga, and R. V. de Moraes, "Implications of the application of recursive least squares algorithms to satellite orbit determination using GPS measurements," WSEAS Transactions on Systems, vol. 8, no. 3, pp. 334-343, 2009.

[16] G. J. Bierman and C. L. Thornton, "Numerical comparison of Kalman filter algorithms: orbit determination case study," Automatica, vol. 13, no. 1, pp. 23-35, 1977.

[17] R. G. Brown and P. Y. C. Hwang, "A kalman filter approach to precision GPS geodesy," Navigation, vol. 30, no. 4, pp. 338-349, 1983.

[18] A. Gelb, J. F. Kasper Jr., R. A. Nash Jr., C. F. Price, and A. A. Sutherland Jr., Applied Optimal Estimation, The MIT Press, Inglaterra, UK, 1974.

[19] P. S. Maybeck, Stochastic Models, Estimation, and Control, vol. 1, Academic Press, New York, NY, USA, 1979.

[20] J. R. Raol and N. K. Sinha, "On the orbit determination problem," IEEE Transactions on Aerospace and Electronic Systems, vol. 21, no. 3, pp. 274-291, 1985.

[21] T. P. Yunck, "Orbit determination," in Global Positioning System: Theory and Applications, vol. 2, chapter 21, pp. p559-p592, AIAA, 1996.

[22] O. Montenbruck and E. Gill, Satellite Orbits: Models, Methods, and Applications, Springer, New York, NY, USA, 2001.

[23] H. K. Kuga, Adaptive estimation of orbits applied to low altitude satellites [M.S. thesis], INPE, São José dos Campos, Brazil, 1982, INPE-2316-TDL/079, in Portuguese.

[24] H. K. Kuga, Orbit determination of Earth artificial satellites through state estimation techniques combined with smoothing techniques [Ph.D. dissertation], INPE, São José dos Campos, Brazil, 1989, INPE-4959-TDL/079, in Portuguese.

[25] A. P. M. Chiaradia, H. K. Kuga, and A. F. B. A. Prado, "Single frequency GPS measurements in real-time artificial satellite orbit determination," Acta Astronautica, vol. 53, no. 2, pp. $123-$ 133, 2003.

[26] S. Pines, "Uniform representation of the gravitational potential and its derivatives," AIAA Journal, vol. 11, no. 11, pp. 1508-1511, 1973.

[27] A. P. M. Chiaradia, H. K. Kuga, and A. F. B. A. Prado, "Investigation on ionospheric correction models for GPS signals," in Advances in Space Dynamics, A. F. B. A. Prado, Ed., pp. 214-219, Instituto Nacional de Pesquisas Espaciais, 2000.

[28] A. P. M. Chiaradia, E. Gill, O. Montenbruck, H. K. Kuga, and A. F. B. A. Prado, Algorithms for on-Board Orbit Determination 
Using GPS-OBODE-GPS, DLR, Alemanha, Germany, 2000, DLR-GSOC TN 00-04.

[29] A. Leick, GPS Satellite Surveying, INC, John Wiley \& Sons, 2nd edition, 1995.

[30] A. P. M. Chiaradia, H. K. Kuga, and A. F. B. A. Prado, "Investigation of simplified models for orbit determination using single frequency GPS measurements," Journal of the Brazilian Society of Mechanical Sciences, vol. 21, pp. 165-172, 1999.

[31] H. K. Kuga, Transition Matrix of the Elliptical Keplerian Motion, INPE, São José dos Campos, Brazil, 1986, INPE-3779-NTE/250, in Portuguese.

[32] F. L. Markley, "Approximate cartesian state transition matrix," The Journal of the Astronautical Sciences, vol. 34, no. 12, pp. 161$169,1986$.

[33] A. P. M. Chiaradia, H. K. Kuga, and A. F. B. A. Prado, "Comparison between two methods to calculate the transition matrix of orbit motion," Mathematical Problems in Engineering, vol. 2012, Article ID 768973, 12 pages, 2012.

[34] V. M. Gomes, H. K. Kuga, and A. F. B. A. Prado, "Real time orbit determination with different force fields," WSEAS Transactions on Applied and Theoretical Mechanics, vol. 5, no. 1, pp. 23-32, 2010.

[35] V. M. Gomes, A. F. B. A. Prado, and H. K. Kuga, "Low thrust maneuvers for artificial satellites," WSEAS Transactions on Applied and Theoretical Mechanics, vol. 3, no. 10, pp. 859868, 2008.

[36] V. M. Gomes, Prado, A. F. B. A, and H. K. Kuga, "Filtering the GPS navigation solution for real time orbit determination using different dynamical models," in Proceedings of the 5th WSEAS International Conference on Applied and Theoretical Mechanics (MECHANICS '09), vol. 1, pp. 1-10, Puerto de La Cruz, Spain, 2009.

[37] H. W. Press, B. P. Flannery, S. A. Teukolsky, and W. T. Vetterling, Numerical Recipesedition, Cambridge University Press, 3rd edition, 1987. 


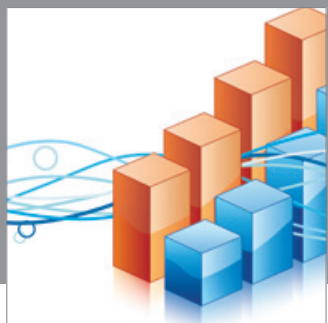

Advances in

Operations Research

mansans

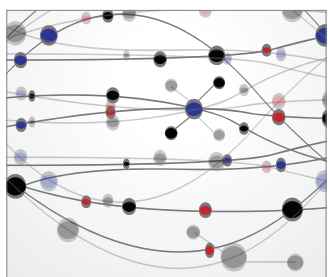

The Scientific World Journal
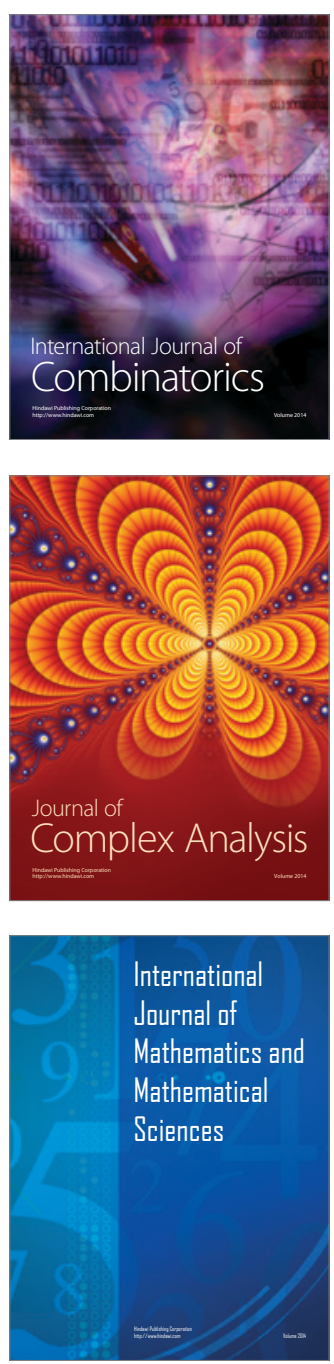
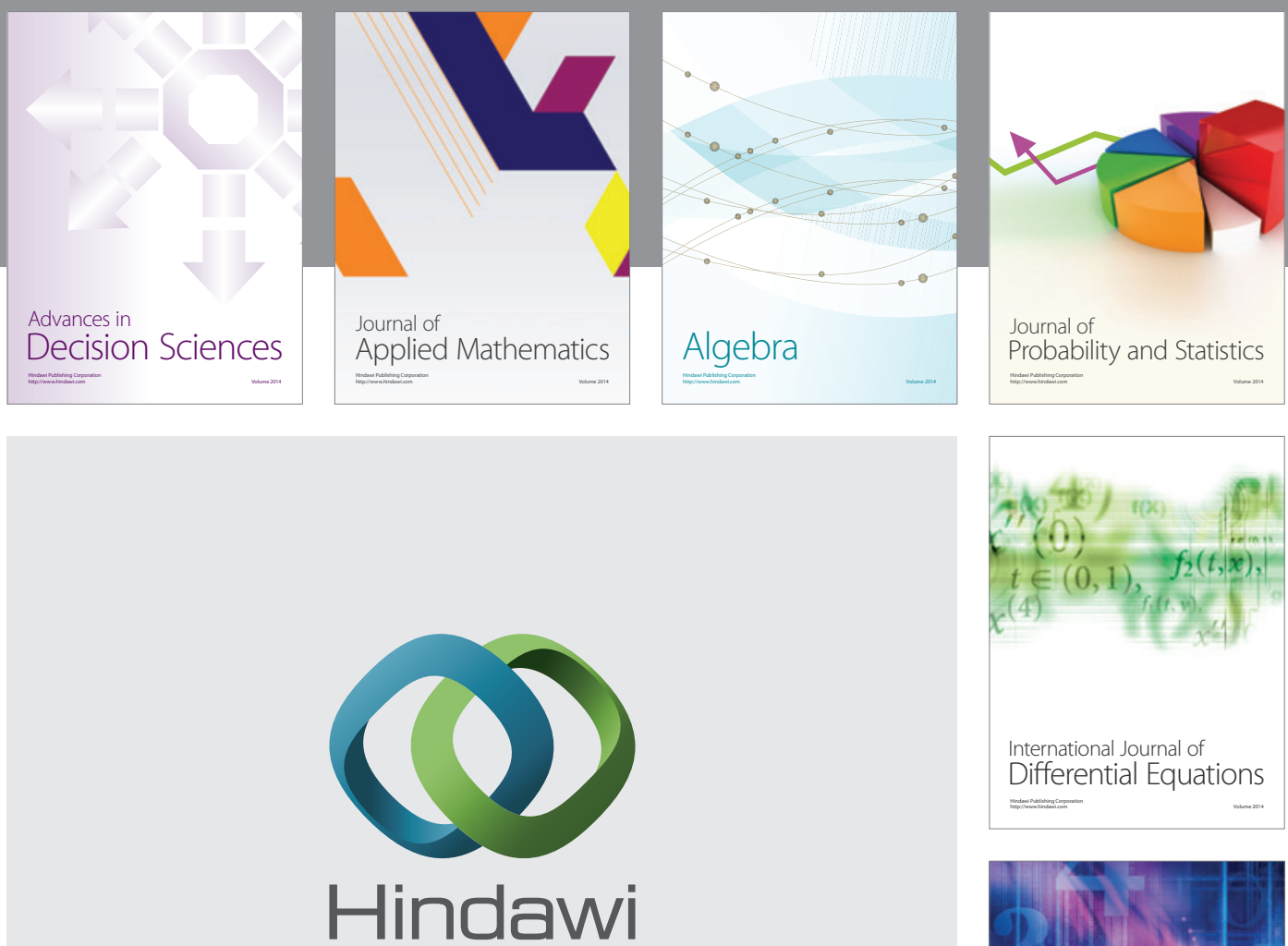

Submit your manuscripts at http://www.hindawi.com
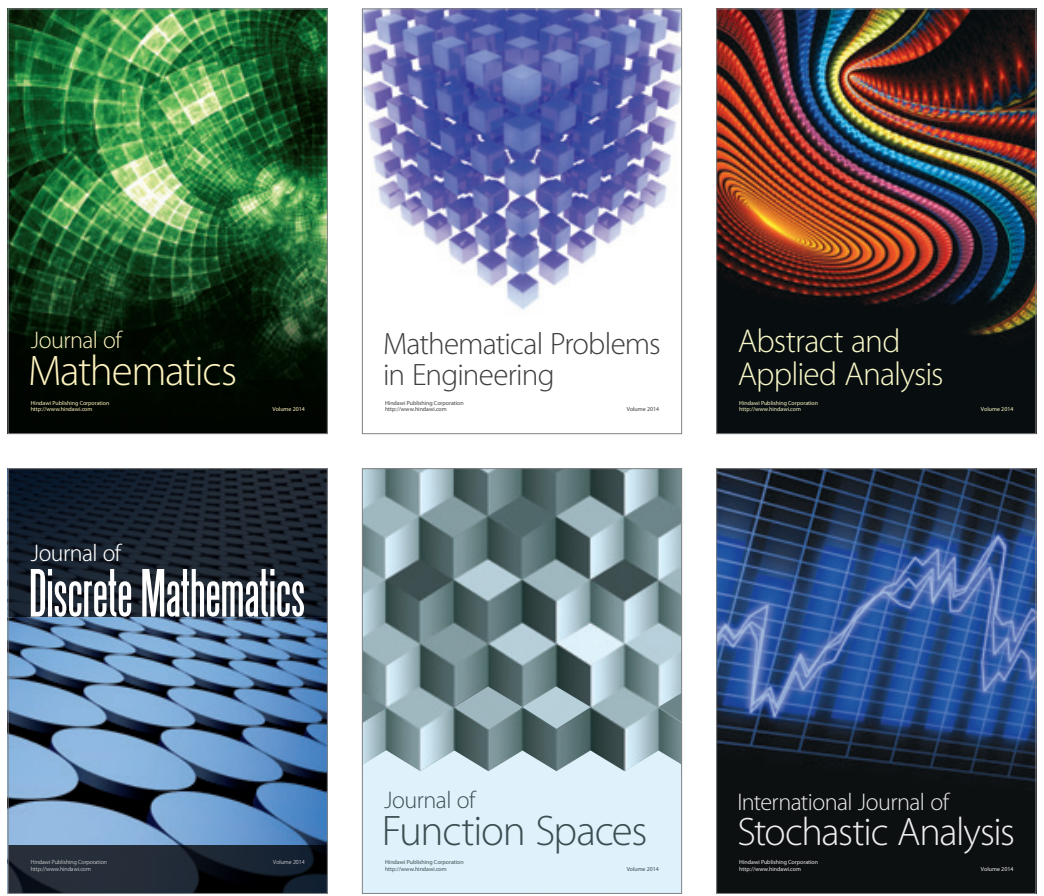

Journal of

Function Spaces

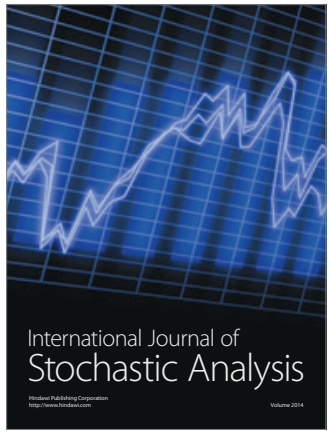

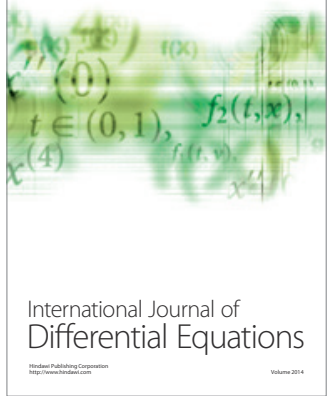
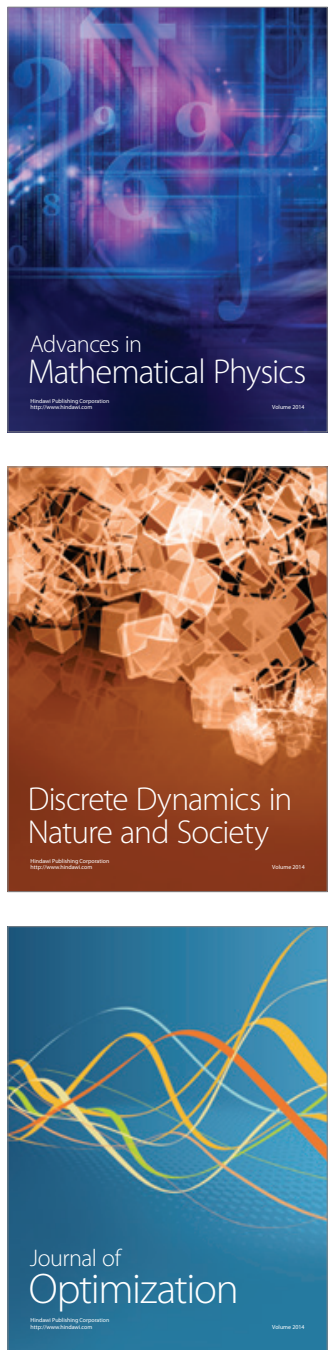